Case Report:

\title{
Chondrosarcoma of Cranial Vault: Case Report and Review of Literature
}

\author{
Keshav Mishra ${ }^{1}$, Somnath Sharma ${ }^{1}$, Devendra K. Purohit ${ }^{1^{*}}$, , Arpita Jindal $^{2} \mathrm{Q}$ \\ 1. Department of Neurosurgery, SMS Medical College, Rajasthan, India \\ 2. Department of Pathology, SMS Medical College, Rajasthan, India
}

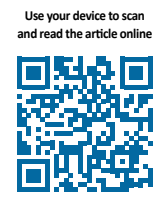

Citation: Mishra K, Sharma S, K. Purohit D, Jindal A. Chondrosarcoma of Cranial Vault: Case Report and Review of Literature. Iran J Neurosurg. 2021; 7(2):119-124. http://dx.doi.org/10.32598/irjns.7.2.7

http://dx.doi.org/10.32598/irjns.7.2.7

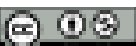

Article info:

Received: 08 Mar 2021

Accepted: 29 Mar 2021

Available Online: 01 Apr 2021

Keywords:

Brain neoplasm, Brain tumor

\section{ABSTRACT}

Background and Importance: Chondrosarcoma is a rare malignant cartilage forming tumor, usually arising from long bones and rarely observed in the cranium. In the cranium, it commonly arises from the skull base with the skull vault being a highly unusual site.

Case Presentation: We present the case of a 30-year female presenting with complaints of headache for 6 months. The patient was found to have large chondrosarcoma in the right frontoparietal region, which is an extremely rare location. The final diagnosis was based on histological examination, suggestive of well-differentiated chondrosarcoma [the World Health Organization (WHO); grade I].

Conclusion: Cranial vault chondrosarcoma is an uncommon histological diagnosis with surgery as the primary treatment option; however, adjuvant radiotherapy plays a definitive role, especially in aggressive or incomplete removed cases.

\section{"Corresponding Author:}

Devendra K. Purohit, MD.

Address: Department of Neurosurgery, SMS Medical College Jaipur, Rajasthan, India

Tel: +91 (98) 11052547

E-mail: devendrapurohit@rediffmail.com 


\section{Highlights}

- Chondrosarcoma is a malignant cartilaginous tumor, rarely seen in the cranium mostly in the skull base.

- The Chondrosarcoma of the skull vault is a rare entity.

- Radiological findings are suggestive but definitive diagnosis is based on histological analysis.

- Surgery is the mainstay of treatment followed by adjuvant radiotherapy to reduce recurrences.

- Prognosis is based on histological type and grade, the extent of tumor removal, using the post-operative adjuvant radiation therapy, and history of previous treatment.

\section{Plain Language Summary}

Chondrosarcoma is a malignant cartilage-forming tumor seen usually in long bones and rarely in the cranium with the skull base being its preferred location. We are reporting a case of a 30-year female patient who presented to us with a headache of 6-month duration who was found to have a large tumor in the right frontal region of the calvarium. She underwent surgery and gross total tumor excision was done and histology showed chondrosarcoma (WHO Grade 1). She was referred to radiotherapy despite complete resection to reduce the risk of recurrence.

\section{Background and Importance}

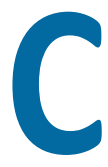

hondrosarcomas are cartilage, producing slow-growing malignant tumors. They primarily affect the long bones of extremities and pelvis, generally sparing the cranium. Primary chondrosarcoma comprises $0.16 \%$ of intracranial neoplasm [1]. The skull base is the most common intracranial location with the petrous bone and sphenoid bone being the most common sites of origin [2], although the cranial vault is less commonly affected. Chondrosarcoma arises from residual embryonic chondrogenic cells residing along synchondrosis or mesenchymal stem cells abnormally localized into the cranial vault bones during intramembranous ossification [3-5]. Chondrosarcomas are classified according to histopathology into conventional (producing hyaline, or myxoid cartilage, or both), dedifferentiated, clear cell, and mesenchymal type [6] with conventional subtype having a better prognosis. The World Health Organization (WHO) has categorized chondrosarcoma into 3 grades. It also has prognostic significance, as follows: grade I: well-differentiated, grade II: moderately differentiated, and grade III: poorly differentiated.

However, a malignant tumor, chondrosarcoma, has a slow growth rate and metastasizes late in the course of the disease. It presents with the symptoms of invasion and mass effect on adjacent neural structures; thus, headache and diplopia are the most common present- ing symptoms. Radiological studies facilitated the diagnosis of intracranial chondrosarcoma; however, due to the lack of diagnostic criteria and the rarity of the lesion, pre-operative diagnosis is a rarity. Therefore, pathological diagnosis forms the gold standard. Radical excision of the lesion with the maximal possible preservation of adjacent vital neural structures with post-operative radiation therapy for the residual is the standard of care [7-9].

\section{Case Presentation}

A 30-year old female patient presented to our neurosurgery department with the complaint of a 6-month headache which had increased in intensity in the last month. There was no significant history of head trauma or family history of malignancy. There was no history of pain or swelling in any part of the patient's body. On neurological examination, the patient was conscious and oriented without the signs of focal neurological deficits (e.g. motor weakness, visual deficits, or abnormalities of higher mental function) or the presence of pathological reflexes, like the Babinski sign. There was no visible or palpable swelling or lumps evident on examination. Brain Magnetic Resonance Imaging (MRI) was suggestive of well-defined extra-axial (as evidenced by the presence of CSF cleft around the lesion with vascular flow voids and the buckling of surrounding brain tissue) heterogenous intensity lesion in the right frontoparietal lobe, i.e., reflected mild hyperintensity with hyperintense center and peripheral flow voids on $\mathrm{T} 2 \mathrm{~W}$ 


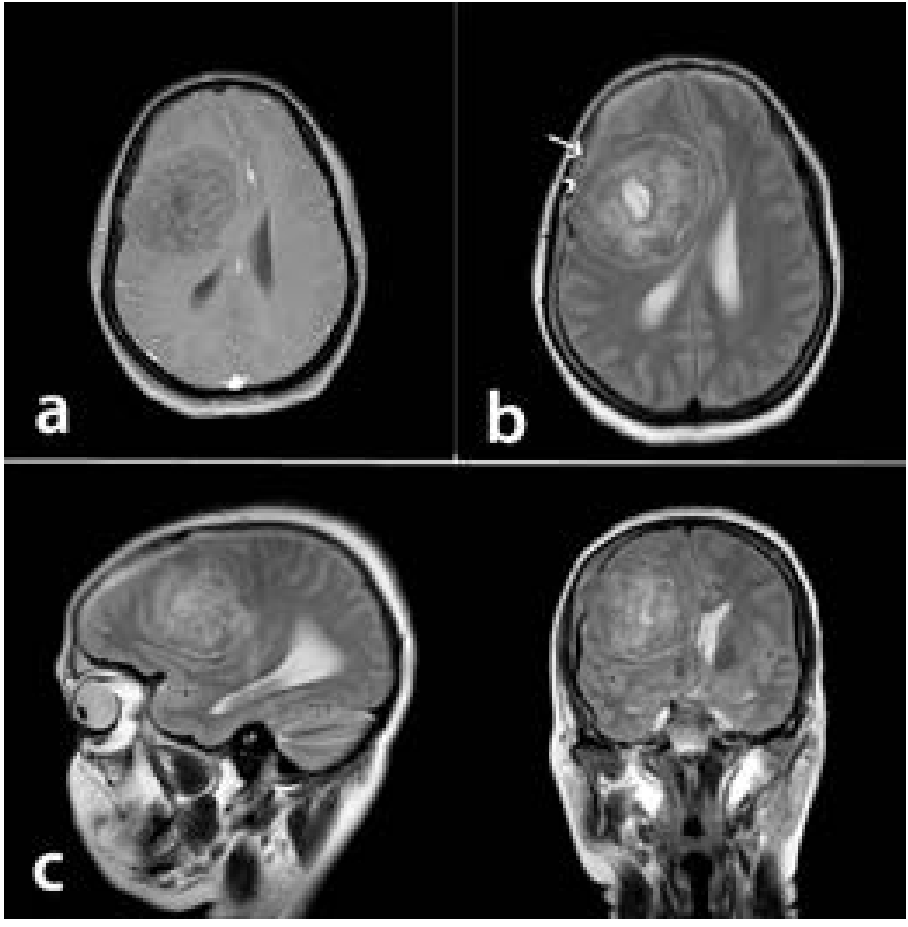

Figure 1. MRI brain, suggestive of well-defined extra-axial heterogenous intensity lesion in right frontoparietal lobe presenting mild hyperintensity with hyperintense center and peripheral flow voids on T2W sequence (arrow showing changes in the intensity of surrounding bone; arrowhead illustrating continuity between the lesion and the bone demonstrating the same attenuation).

sequence (Figure 1). Moreover, T1W images showed a hypointense lesion, demonstrating diffusion restriction on diffusion-weighted imaging. The lesion showed minimal contrast enhancement with no sign of dural enhancement or dural tail. The patient underwent left frontoparietal craniotomy and intraoperatively; the mass was greyish white, stony-hard, avascular, and attached to overlying bone. The gross total excision of the lesion was performed along with the involved bone.

Post-operatively, the reported patient was neurologically intact and the post-operative imaging confirmed the total excision of the intracranial mass lesion. The patient had an uneventful hospital stay and was discharged on the seventh post-operative day without neurological deficits.

On histopathological examination, the tumor indicated increased cellularity with abundant blue-gray cartilaginous matrix and the lobules of cartilage (Figure 2). The chondrocytes were atypical, varying in size and shape, and contained enlarged hyperchromatic nuclei, suggestive of well-differentiated chondrosarcoma (the WHO, grade I). The studied patient was subsequently referred for post-operative radiotherapy; the patient was symptom-free and recurrence-free at the 10-month followup examination.

\section{Discussion}

Chondrosarcoma is the second most common primary malignant cartilage, forming the tumor of bone. It mainly presents in the fourth to fifth decades (classical type) of life; however, more malignant variants present at earlier ages. Males are affected twice as commonly as females. Even though the majority of intracranial CSA (Chondrosarcoma) arise de novo; however, they can also be observed in association with Ollier's disease, Maffucci syndrome, Paget's disease, and osteochondroma.

It mainly affects the axial skeleton, especially the pelvis, shoulders, and ribs. Intracranial chondrosarcoma, first reported by Mott in 1899 [10] is not a common occurrence with the skull base being the most frequently affected intracranial site and no gender inequality. Chondrosarcoma arising from the cranial vault is an even rarer entity [1]. In a systematic review published by Bloch et al. in 2009 , clivus (32\%) was found to be the most common site of origin, followed by the temporooccipital junction [11] in this regard (27\%). However, Corten et al. reported petrous bone (37\%) to be the most common site, followed by occipital-clival (23\%), and sphenoid bone (20\%). Other uncommon locations have also been reported, such as brain parenchyma and choroid plexus [12]. 


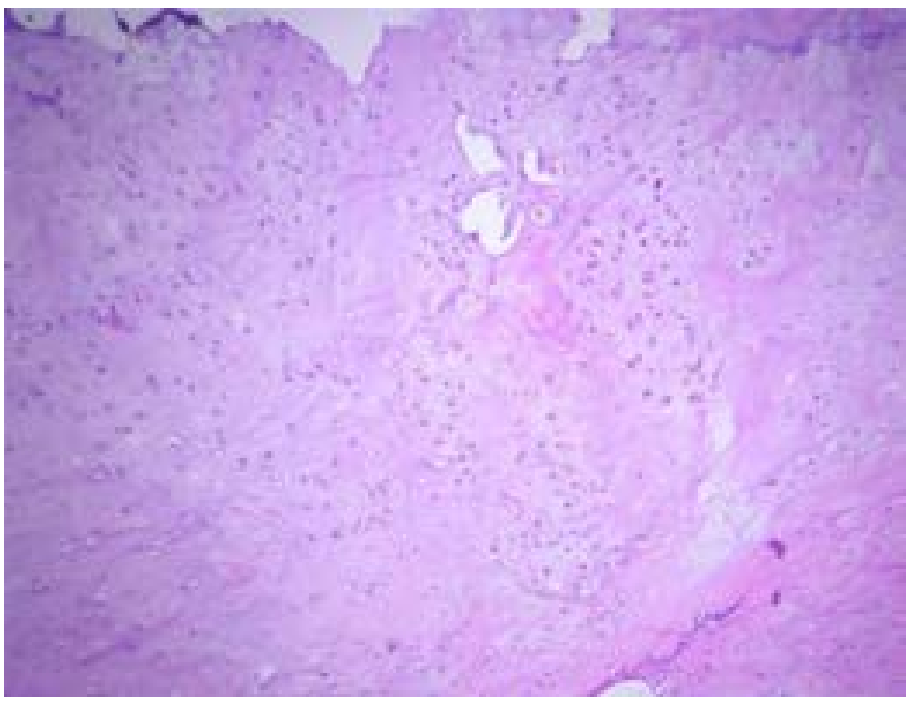

Figure 2. Histopathological examination, reflecting the tumor with increased cellularity, abundant blue-gray cartilaginous matrix, and the lobules of cartilage

Intracranial CSA has been divided into 3 types according to histopathology; well-differentiated or classical type; myxoid type, and mesenchymal type. Korten reported the classical variety $(62 \%)$ to be the commonest; however, myxoid and mesenchymal variants formed $8 \%$ and $30 \%$ of the distribution. Mesenchymal subtype forms the majority of lesions arising above the skull base 4 and a predilection for young patients, aged 10-30 years.

Multiple theories have been proposed to explain the origin of cartilage-based lesions within the skull. Although the bones of the cranial vault develop predominantly by intramembranous ossification, endochondral ossification is also responsible for the development of several sites in the mature skull, including a large part of the petrous portion of the temporal bone, the areas of the petro-occipital, the spheno-occipital, and the spheno-petrosal synchondrosis. Intracranial chondrosarcomas might develop from the chondrocytes within the rest of the endochondral cartilage that may be present in these areas. The theory by Lee et al. postulates that meninges may contain multipotent mesenchymal cells that can give rise to cartilage forming tumors [13]; however, Alpers believed the meningeal fibroblasts to be the culprit cells. Most CSA is located in the skull base; thus, they are believed to arise from cartilage forming the synchondroses in the bones of the skull base [14, 15]

Computed Tomography (CT) scan in intracranial CSA usually indicates the presence of isodense to hyperdense mass, reflecting heterogeneous enhancement and calcification [16]. On MRI, CSA appears to be T1 hypointense; however, it demonstrates a high signal on
T2 weighted images. Most classical and some mesenchymal variety CSA present decreased vascularity; however, intense contrast enhancement has been reported in both low grade and mesenchymal varieties $[17,18]$.

Radical tumor excision is the standard of care for intracranial CSA; however, surgery alone has been associated with a recurrence rate as high as $44 \%$ over 5 years, which can be brought down by the addition of postoperative radiation therapy. Blotch et al. demonstrated the benefit of adjuvant post-operative radiation therapy which reduced the 5 -year mortality to $4 \%$, compared to $26 \%$ in the group treated by surgery alone. The use of adjuvant radiation therapy has also been recommended by other studies $[19,20]$. Adjuvant radiation therapy has been recommended by some scholars, even after radical tumor resection [21].

Numerous studies have highlighted the survival benefit of adjuvant radiotherapy; however, there is no grade I recommendation for the same. Some authors have speculated that low-grade CSA undergoing complete surgical resection presents good survival without the addition of postoperative adjuvant radiation [19]. Rosenberg examined 200 patients of the skull base CSA, treated in a single institution with a combination of surgery and fractionated proton beam radiation. Accordingly, they reported $99 \%$ of 5-year as well as 10 -year disease-specific survival rates. However, other large series failed to achieve similar levels of success with Bloch et al. reporting an $89 \%$ overall survival rate in their series with average survival rates of 53.7 months [13]. In this case, the tumor was located at the left frontoparietal 
region, and the total surgical excision of the tumor was achieved; subsequently, the patient was referred for radiotherapy with a favorable overall prognosis.

The prognosis of extracranial CSA is worse than their cranial counterparts with survival rates approaching as low as $41 \%$ [22]. Numerous studies have found the overall 5-year survival rates to be $85 \%-100 \%[23,24]$. Blotch, in his case series study, found an average survival of 53.7 months in intracranial CSA cases.

The prognosis of intracranial CSA is determined by several factors, including histological type and grade, the extent of tumor removal, the use of postoperative adjuvant radiation therapy, and a history of previous treatment. The mesenchymal type has the most dismal outcome with 5 -year mortality as high as $54 \%$, while the classical subtype presented a 6\% 5-year mortality [19]. The 5-year survival for chondrosarcoma grades I, $\mathrm{II}$, and III have been reported to be $95 \%, 90 \%$, and $75 \%$ respectively by Blotch and associates [11]. Evans et al. have similarly reported a 5 -year survival rate of chondrosarcomas grades I, II, to be $90 \%$ and $81 \%$; however, for grade III tumors, it decreased to $43 \%$, compared to $75 \%$ in the aforementioned study [25].

The literature on the effect of previous treatment modalities concerning the prognosis of CSA provided heterogeneous results. Tzortzidis et al. reported decreased tumor-free survival in patients requiring multiple surgeries [26]. However, Oghalai and Blotch et al. found no significant correlation between mortality and a history of previous treatments for CSA.

\section{Conclusion}

Intracranial chondrosarcoma, especially those arising from the cranial vault is a rare malignant cartilage-producing tumor. The preoperative diagnosis of intracranial chondrosarcoma can be challenging. This is because of its rare occurrence and similarity in clinical and radiological presentation with other intracranial pathologies, like meningioma. Thus, histopathology forms the mainstay of diagnosis and provides valuable prognostic information. It suggests high rates of local recurrence after surgical excision alone; as a result, radiotherapy is an essential adjunctive treatment, increasing the recurrencefree disease interval and overall survival rates.

\section{Ethical Considerations}

\section{Compliance with ethical guidelines}

There were no ethical considerations to be considered in this research.

Funding

This research did not receive any grant from funding agencies in the public, commercial, or non-profit sectors.

\section{Authors' contributions}

Conception and design: Keshav Mishra, Somnath Sharma, Devendra K. Purohit; Data collection: Keshav Mishra; Data analysis and interpretation: Keshav Mishra, Somnath Sharma, Devendra K. Purohit, Arpita Jindal; Drafting the article: Keshav Mishra, Somnath Sharma; Critically revising the article: Keshav Mishra, Somnath Sharma, Devendra K. Purohit, Arpita Jindal; Reviewing submitted version of manuscript: Keshav Mishra, Somnath Sharma, Devendra K. Purohit, Arpita Jindal; Approving the final version of the manuscript: Keshav Mishra, Somnath Sharma, Devendra K. Purohit, Arpita Jindal.

\section{Conflict of interest}

The authors declared no conflict of interest.

\section{References}

[1] Berkmen YM, Blatt ES. Cranial and intracranial cartilaginous tumours. Clinical Radiology. 1968; 19(3):327-33. [DOI:10.1016/S0009-9260(68)80019-4] [PMID]

[2] Healey JH, Lane J. Chondrosarcoma. Clinical Orthopaedics and Related Research. 1986; 204:119-29. [DOI:10.1097/00003086-198603000-00012]

[3] Ryzewicz M, Manaster BJ, Naar E, Lindeque B. Low-grade cartilage tumors: Diagnosis and treatment. Orthopedics. 2007; 30(1):35-46. [DOI:10.3928/01477447-20070101-08] [PMID]

[4] Korten AGGC, ter Berg HJW, Spincemaille GH, van der Laan RT, Van de Wel AM. Intracranial chondrosarcoma: Review of the literature and report of 15 cases. Journal of Neurology, Neurosurgery, and Psychiatry. 1998; 65(1):88-92. [DOI:10.1136/jnnp.65.1.88] [PMID] [PMCID]

[5] Bloch OG, Jian BJ, Yang I, Han SJ, Aranda D, Ahn BJ, et al. Cranial chondrosarcoma and recurrence. Skull Base. 2010 20(3):149-56. [DOI:10.1055/s-0029-1246218] [PMID] [PMCID] 
[6] Wanebo JE, Bristol RE, Porter RR, Coons SW, Spetzler RF. Management of cranial base chondrosarcomas. Neurosurgery. 2006; 58:249-55. [DOI:10.1227/01.NEU.0000194834.74873.FB]

[7] Austin-Seymour M, Munzenrider J, Goitein M, Verhey L, Urie M, Gentry R, et al. Fractionated proton radiation therapy of chordoma and low-grade chondrosarcoma of the base of the skull. Journal of Neurosurgery. 1989; 70(1):13-7. [DOI:10.3171/ jns.1989.70.1.0013] [PMID]

[8] Gay E, Sekhar LN, Rubinstein E, Wright DC, Sen C, Janecka IP, et al. Chordomas and chondrosarcomas of the cranial base: Results and follow-up of 60 patients. Neurosurgery. 1995; 36(5):887-97. [DOI:10.1227/00006123-199505000-00001] [PMID]

[9] Sen CN, Sekhar LN, Schramm VL, Janecka IP. Chordoma and chondrosarcoma of the cranial base: An 8-year experience. Neurosurgery. 1989; 25(6):931-41. [DOI:10.1227/00006123198912000-00013] [PMID]

[10] Mott FW. Chondrosarcoma springing from the sella turcica. Archives of Neurology and Psychiatry. 1899; 1:432-3. https:/ / scholar.google.com/scholar?hl=fa\&as_sdt $=0 \% 2 \mathrm{C} 5 \& \mathrm{q}=\mathrm{Chon}$ drosarcoma+springing+from+the+sella+turcica\&btnG=

[11] Bloch OG, Jian BJ, Yang I, Han SJ, Aranda D, Ahn BJ, et al. A systematic review of intracranial chondrosarcoma and survival. Journal of Clinical Neuroscience. 2009; 16(12):1547-51. [DOI:10.1016/j.jocn.2009.05.003] [PMID] [PMCID]

[12] Güneș M, Günaldi O, Tuğcu B, Tanriverdi O, Güler AK, Cöllüoğlu B. Intracranial chondrosarcoma: A case report and review of the literature. Minimally Invasive Neurosurgery. 2009; 52(5-6):238-41. [DOI:10.1055/s-0028-1128117] [PMID]

[13] Lee YY, Van Tassel P, Raymond AK. Intracranial dural chondrosarcoma. American Journal of Neuroradiology. 1988; 9(6):1189-93. [PMID] [PMCID]

[14] Gabrielsen TO, Kingman Jr AF. Osteocartilaginous tumors of the base of the skull. Report of a unique case and review of the literature. The American Journal of Roentgenology, Radium Therapy, and Nuclear Medicine. 1964; 91:1016-23. [PMID]

[15] Sarwar M, Swischuk LE, Schechter MM. Intracranial chondromas. American Journal of Roentgenology. 1976; 127(6):973-7. [DOI:10.2214/ajr.127.6.973] [PMID]

[16] Erdem E, Angtuaco EC, Van Hemert R, Park JS, Al-Mefty O. Comprehensive review of intracranial chordoma. Radiographics. 2003; 23(4):995-1009. [DOI:10.1148/rg.234025176]

[17] Gerszten PC, Pollack IF, Hamilton RL. Primary parafalcine chondrosarcoma in a child. Acta Neuropathologica. 1998, 95(1):111-4. [DOI:10.1007/s004010050773] [PMID]

[18] Salazar J, Vaquero J, Aranda JF, Menendez J, Jimenez MD, Bravo G. Choroid plexus papilloma with chondroma: Case report. Neurosurgery. 1986; 18(6):781-3. [DOI:10.1227/00006123198606000-00018] [PMID]

[19] Isaacson B, Kutz JW, Roland PS. Lesions of the petrous apex: Diagnosis and management. Otolaryngologic Clinics of North America. 2007; 40(3):479-519. [DOI:10.1016/j.otc.2007.03.003] [PMID]

[20] Neff B, Sataloff RT, Storey L, Hawkshaw M, Spiegel JR. Chondrosarcoma of the skull base. The Laryngoscope. 2002 112(1):134-9. [DOI:10.1097/00005537-200201000-00023] [PMID]
[21] Bosma JJ, Kirollos RW, Broome J, Eldridge PR. Primary intradural classic chondrosarcoma: Case report and literature review. Neurosurgery. 2001; 48(2):420-3. [DOI:10.1097/00006123-200102000-00038] [PMID]

[22] McLoughlin GS, Sciubba DM, Wolinsky JP. Chondroma/ chondrosarcoma of the spine. Neurosurgery Clinics of North America. 2008; 19(1):57-63. [DOI:10.1016/j.nec.2007.09.007] [PMID]

[23] Oghalai JS, Buxbaum JL, Jackler RK, McDermott MW. Skull base chondrosarcoma originating from the petroclival junction. Otology \& Neurotology. 2005; 26(5):1052-60. [DOI:10.1097/01.mao.0000185076.65822.f7] [PMID]

[24] Colli BO, Al-Mefty O. Chordomas of the skull base: Followup review and prognostic factors. Neurosurgical Focus. 2001; 10(3):E1. [DOI:10.3171/foc.2001.10.3.2] [PMID]

[25] Evans HL, Ayala AG, Romsdahl MM. Prognostic factors in chondrosarcoma of bone: A clinicopathologic analysis with emphasis on histologic grading. Cancer. 1977; 40(2):818-31. [DOI:10.1002/1097-0142(197708)40:2<818::aidcncr2820400234>3.0.co;2-b] [PMID]

[26] Tzortzidis F, Elahi F, Wright DC, Temkin N, Natarajan SK, Sekhar LN. Patient outcome at long-term follow-up after aggressive microsurgical resection of cranial base chondrosarcomas. Neurosurgery. 2006; 58(6):1090-8. [DOI:10.1227/01. NEU.0000215892.65663.54] [PMID] 